\title{
Cellular Uptake of Fibroin Micro/Nanoparticles as a Method for Three-Dimensional Culture under Magnetic Levitation Conditions
}

\author{
Jae Kwon Shim, Won Hur \\ Department of Bioengineering and Technology, Kangwon National University \\ 192-1 Hyoja-dong, Chuncheon, Korea \\ shim3936@naver.com; wonhur@kangwon.ac.kr
}

\section{Extended Abstract}

Fibroin is the structural protein of silk fibres that has been used for suture materials for decades [1]. Core-shell structured micro/nanoparticles of submicron size were prepared from fibroin without any chemical cross-linking [2]. Fibroin-shell microspheres with a fluorescent core were used to evaluate cellular uptake and to trace intracellular trafficking in murine 3T3 cells [3]. Animal cells that ingested $\mathrm{Fe}_{3} \mathrm{O}_{4}$-containg microspheres have been used to prepare a scaffold-free three-dimensional (3D) culture of animal cells [4, 5]. In this report, we further investigated fibroin micro/nanosphere uptake and multicellular aggregate formation using a variety of cells and cell lines.

The percentage of cells that ingested fibroin microspheres varied significantly depending on the cells used. The efficiency of microsphere uptake was in the order of human mesenchymal stem cell, murine fibroblast 3T3, monocyte Raw264.7, murine melanocyte B16F10 and human dermal fibroblast. Disk- or spheroid- shaped multicellular aggregates were obtained by magnetic levitation of cells ingested $\mathrm{Fe}_{3} \mathrm{O}_{4}$-containg microspheres. A disk-shaped multicellular surface culture up to $15 \mathrm{~mm}$ in diameter was obtained using B16F10 cells and a spheroid up to $3.5 \mathrm{~mm}$ in diameter was made from $3 \mathrm{~T} 3$ cells. Microscopic study showed that $\mathrm{Fe}_{3} \mathrm{O}_{4}$-containg microspheres aligned parallel to the magnetic field and that a necrotic core was developed in 3T3 spheroids possibly due to diffusion limitation of nutrient or oxygen. Micro CT scan also revealed that $\mathrm{Fe}_{3} \mathrm{O}_{4}$ distribution was not uniform throughout the spheroid. While, B16F10 and Raw264.7 cells showed surface growth at the air-medium interface, which is hardly observed in animal cell cultures.

The present approach produced millimetre-scale multicellular spheroids or multilayer cell cultures depending on cells used, which has a potential being developed to surrogate tissue for toxicant monitoring, for extracellular matrix synthesis and for differentiation study.

\section{References}

[1] G. H. Altman, "Silk-based biomaterials," Biomaterials, vol. 24, no. 3, pp. 401-416, 2003.

[2] E. J. Kim, "Preparation of core-shell microcapsules using nanodispersed fibroin," J. Appl. Poly. Sci., vol. 121, no. 6, pp. 3460-3465, 2011.

[3] E. J. Go, "In vitro cellular uptake of fibroin microspheres and its dependency on the cell cycle stage," $J$. microencapsul., vol. 30, no. 2, pp. 124-131, 2013.

[4] J. H. Lee, "Scaffold-free formation of a millimeter-scale multicellular spheroid with an internal cavity from magnetically levitated 3T3 cells that ingested iron oxide-containing microspheres," Biotechnol. Bioeng., vol. 111, no. 5, pp. 1038-1047, 2014.

[5] Y. G. Jeong, "A scaffold-free surface culture of B16F10 murine melanoma cells based on magnetic levitation," Cytotechnology, vol. 68, no. 6, pp. 2323-2334, 2016. 